\title{
Comparative genomics of whole-cell pertussis vaccine strains from India
}

\author{
Shweta Alai ${ }^{1}$, Vikas C. Ghattargi ${ }^{2}$, Manish Gautam ${ }^{3}$, Krunal Patel ${ }^{3}$, Shrikant P. Pawar ${ }^{2}$, Dhiraj P. Dhotre², \\ $U_{m e s h}$ Shaligram ${ }^{3}$ and Sunil Gairola ${ }^{3^{*}}$
}

\begin{abstract}
Background: Despite high vaccination coverage using acellular (ACV) and whole-cell pertussis (WCV) vaccines, the resurgence of pertussis is observed globally. Genetic divergence in circulating strains of Bordetella pertussis has been reported as one of the contributing factors for the resurgence of the disease. Our current knowledge of B. pertussis genetic evolution in circulating strains is mostly based on studies conducted in countries using ACVs targeting only a few antigens used in the production of ACVs. To better understand the adaptation to vaccine-induced selection pressure, it will be essential to study B. pertussis populations in developing countries which are using WCVs. India is a significant user and global supplier of WCVs. We report here comparative genome analyses of vaccine and clinical isolates reported from India. Whole-genome sequences obtained from vaccine strains: WCV (J445, J446, J447 and J448), ACV (BP165) were compared with Tohama-I reference strain and recently reported clinical isolates from India (BPD1, BPD2). Core genome-based phylogenetic analysis was also performed using 166 isolates reported from countries using ACV.

Results: Whole-genome analysis of vaccine and clinical isolates reported from India revealed high genetic similarity and conserved genome among strains. Phylogenetic analysis showed that clinical and vaccine strains share genetic closeness with reference strain Tohama-I. The allelic profile of vaccine strains (J445:ptxP1/ptxA2/prn1/fim2-1/fim3-1; J446: ptxP2/ptxA4/prn7/fim2-2/fim3-1; J447 and J448: ptxP1/ptXA1/prn1/fim2-1/fim3-1), which matched entirely with clinical isolates (BPD1:ptxP1/ptxA1/prn1/fim2-1 and BPD2: ptxP1/ptXA1/prn1/fim2-1) reported from India. Multi-locus sequence typing (MLST) demonstrated the presence of dominant sequence types ST2 and primitive ST1 in vaccine strains which will allow better coverage against circulating strains of B. pertussis.

Conclusions: The study provides a detailed characterization of vaccine and clinical strains reported from India, which will further facilitate epidemiological studies on genetic shifts in countries which are using WCVs in their immunization programs.
\end{abstract}

Keywords: Bordetella pertussis, Whooping cough, Resurgence, Antigenic variation, Genome organization, Virulence genes, Vaccine-mediated selection

\footnotetext{
* Correspondence: sunil.gairola@seruminstitute.com

${ }^{3}$ Serum Institute of India Pvt. Ltd, Pune, Maharashtra 411028, India

Full list of author information is available at the end of the article
}

(c) The Author(s). 2020 Open Access This article is licensed under a Creative Commons Attribution 4.0 International License, which permits use, sharing, adaptation, distribution and reproduction in any medium or format, as long as you give appropriate credit to the original author(s) and the source, provide a link to the Creative Commons licence, and indicate if changes were made. The images or other third party material in this article are included in the article's Creative Commons licence, unless indicated otherwise in a credit line to the material. If material is not included in the article's Creative Commons licence and your intended use is not permitted by statutory regulation or exceeds the permitted use, you will need to obtain permission directly from the copyright holder. To view a copy of this licence, visit http://creativecommons.org/licenses/by/4.0/ The Creative Commons Public Domain Dedication waiver (http://creativecommons.org/publicdomain/zero/1.0/) applies to the data made available in this article, unless otherwise stated in a credit line to the data. 


\section{Background}

Whooping cough (Pertussis) is a respiratory disease caused by the Gram-negative bacterium Bordetella pertussis [1]. The introduction of whole-cell vaccines (WCVs) in the 1950s and switch to acellular pertussis vaccines (ACVs) targeting a few virulent proteins in the 1990s played a central role in the control of whooping cough [2-5]. In the last decade, despite high vaccination coverage, pertussis has unexpectedly reemerged in several countries [6-12]. Several possible hypotheses were proposed for the resurgence, such as waning of vaccine-induced immunity, improved surveillance and diagnosis of the disease, and genetic divergence among the strains [13-15].

Genetic divergence was mostly studied in circulating strains of $B$. pertussis concerning vaccine antigens such as pertussis toxin ( $\mathrm{ptx}$ ), pertactin (prn), fimbriae (fim) and filamentous hemagglutinin (FHA) [16-19]. The pathogen adaptation in clinical strains was also observed with respect to the emergence of antigen deficient strains [20]. The circulating strains deficient for prn, FHA, and ptx were reported in several countries [2123]. Pertactin deficient strains were first reported in Philadelphia, USA and later found in many countries like France, Japan, Australia, Finland and Italy, where ACVs have been used $[24,25]$. There are also views that adaptation of pertussis strains goes beyond the changes in ACVs associated proteins and involves other virulenceassociated factors and surface-exposed proteins [26]. Strains deficient in tracheal colonization factor (virulence associated protein in B. pertussis), were reported from Belgium, Netherland, and the USA [27]. Besides antigenic divergence, massive gene loss, pseudogenes formation and insertion sequence (IS481) mediated genomic rearrangements are among the significant genomic features of the $B$. pertussis adaptation that became apparent in different comparative genomic studies [28-35]. Recently, a comparative genomic study based on $343 \mathrm{~B}$. pertussis isolates primarily from countries using ACV suggested that adaptive evolution of this pathogen is closely associated with vaccine introduction and emergent strains spread rapidly between countries [28].

Conventional approaches used to study the population of $B$. pertussis include serotyping, genotyping for the key protective antigens and pulsed-field gel electrophoresis (PFGE) [36-38]. While high throughput, these approaches are limited by their sensitivity to detect minor genetic variations within the genome. Whole-genome sequencing of $B$. pertussis isolates and vaccine strains are better suited to understand the impact of vaccination strategies on pathogen diversity. Our current knowledge of $B$. pertussis adaptation is based on studies in countries that are using ACVs. WCVs are based on the use of inactivated whole-cell as an antigen and therefore induce a broader immune response. Thus, to develop effective strategies to prevent pertussis, it is crucial to study $B$. pertussis adaptation globally including countries which are using WCV [35].

WCVs are commonly used in developing countries, and among them, India is the largest global supplier of WCVs and is primarily using WCVs in their immunization program [39-41]. We reported whole genome sequences of $\mathrm{WCV}$ and ACV strains of $B$. pertussis from India $[42,43]$. Genome sequences of two Indian clinical isolates BPD1 (CP034102) and BPD2 (CP034101) are reported. These are the only two isolates reported from India to date [44].

We report here comparative genomic analysis of five vaccine strain and two clinical isolates from India with the reference strain. Phylogenetic analysis of these vaccine strains and isolates was also performed using 166 isolates reported from countries which are using ACV. Such data will provide opportunities for facilitating surveillance of pertussis in India and its comparisons with globally reported trends in $B$. pertussis populations.

\section{Results}

\section{General genome features}

Comparison of general genomic characteristics of five vaccine strains (J445, J446, J447, J448, BP165), and two clinical (BPD1, BPD2) isolates with Tohama-I are summarized in Table 1. Tohama-I have been employed as a reference strain in the study as its genome is completely sequenced and well characterized. Additionally, Tohama-I has been used as a reference in most comparative genomic studies $[33,38]$. The average genome size reported for $B$. pertussis strains is $4.1 \mathrm{Mbp}$, strains $\mathrm{J} 447$ and J448 reported slightly higher size of $4.2 \mathrm{Mbp}$ and $4.3 \mathrm{Mbp}$ respectively. Percent $\mathrm{G}+\mathrm{C}$ for all strains was observed in the range of 67.12 to $67.82 \%$, and gene encoding regions (CDS) were in the range of 3876 to 4128 , which is consistent with the reported values for B. Pertussis strains (Table 1). B. pertussis strains were reported to have genomic deletions and intragenomic rearrangements through IS copy number expansion, predominantly for IS481 ( 250 copies) [30, 33, 38, 46, 47]. The $B$. pertussis genome is reported to have $\sim 238$ copies of IS481, $\sim 17$ copies of IS1663 and $\sim 6$ copies of IS1002. The clinical and vaccine strains copy numbers of IS481, IS1663 and IS1002 were found to be comparable to reported $B$. pertussis genomes.

The insertion of IS elements is known to create pseudogenes in $B$. pertussis genomes [3, 38]. The pseudogenes were also studied in vaccine and clinical isolates as compared to Tohama-I (Table 1). The number of pseudogenes in vaccine strains ranged between 231 to 307, which were lower than Tohama-I. Whereas, clinical isolates pseudogenes ranged from 359 to 384 and were comparable to Tohama-I which reported 359 pseudogenes. 


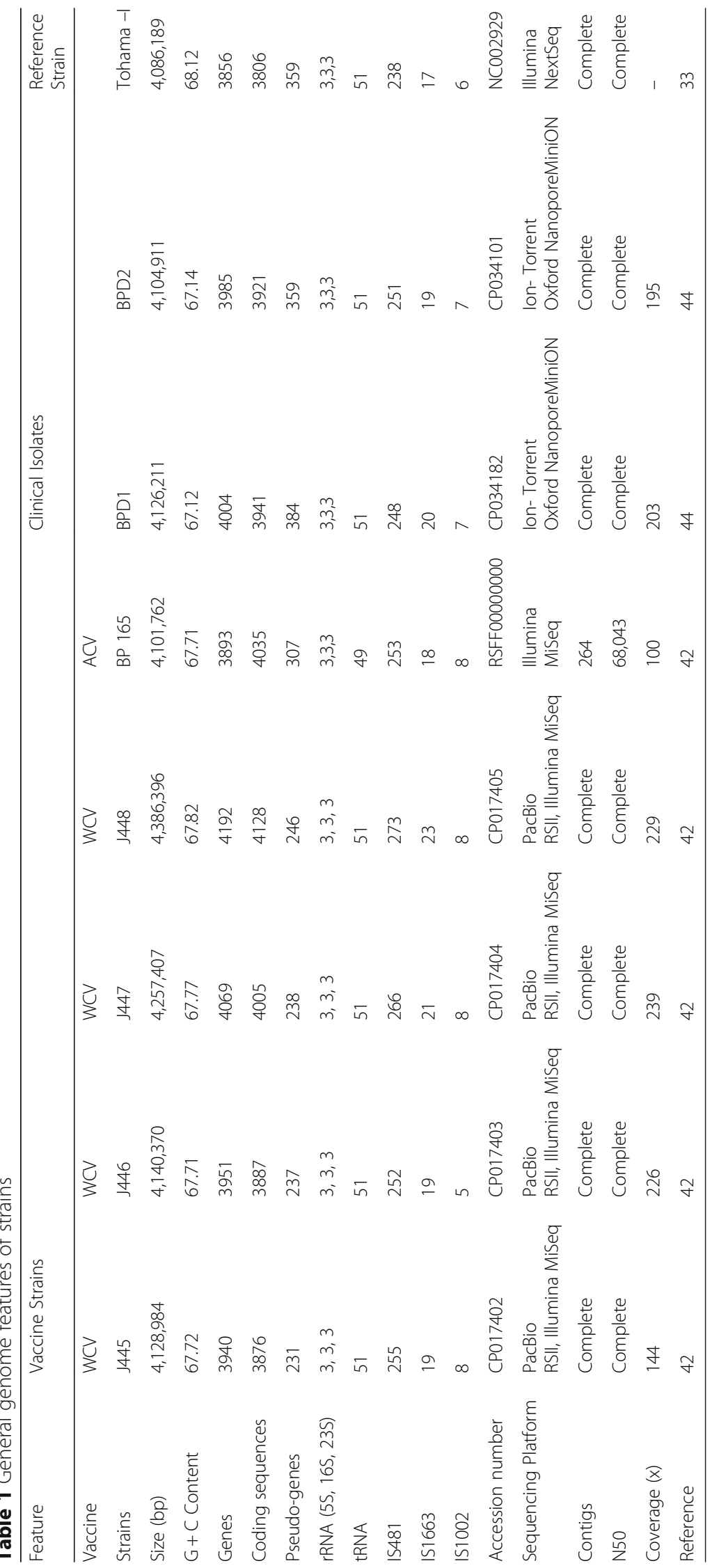


Vaccine and clinical strains genomic similarity (symmetric identity) was assessed using NCBI genome neighbor report (Additional file 1). Vaccine strains and clinical isolates displayed more than $95 \%$ similarity with Tohama-I (Table 2)

\section{Pan-genome analysis}

The pan-genome of 5 vaccines, 2 clinical and 1 reference strain was made up of 3980 genes (Fig. 1). This size is comparable with the pan-genome of $171 \mathrm{~B}$. pertussis strains collected mostly from ACV using countries, which consisted of 3871 genes [49]. The core genome of eight strains consisted of 3070 genes, which constitute approximately $77 \%$ CDSs of these strains. Such high percentage of core genes suggests a low level of genomic diversity among vaccine and clinical strains [50]. The pangenome curve was generated by plotting the total number of distinct gene families against the number of genomes used in this study (Fig. 2). Similarly, the number of shared gene families was plotted against the number of genomes to generate the core-genome plot. BPGA calculates the pan-genome size and core genome size for the given " $\mathrm{N}$ " genomes [49]. The power-law regression model and an exponential curve fit model were calculated for all strains used in this study. Power law regression model suggested as "open but slowly closing pangenomes". The pan-genome model calculated as $\mathrm{y}=$ a.bx ${ }^{c}$ (where a,b,c is parameters) (Fig. 2). Pan-genome size $(\mathrm{n})$ with sequenced genomes $(\mathrm{N})$, was modelled as $\mathrm{n}=\mathrm{kN \gamma}$, where open pan-genome has $\gamma$ value greater than zero and less than one. These lower values signifying a more closed genome with fewer acquired genes. The $\gamma$ value for the classical Bordetella subspecies (0.090) which was lower than that of Bacillus cereus (0.43), indicating the pan-genome is open but slowly closing [50-52]. Previous studies predicted that $B$. anthracis has a closed pan-genome based only on five available genomes $(\alpha=5.6>1)$ [51]. This preliminary

Table 2 Symmetric identity (Genome similarity) between strains based on NCBI genome neighbor report

\begin{tabular}{|c|c|c|c|c|c|c|}
\hline \multirow[t]{2}{*}{ Comparator } & \multicolumn{4}{|c|}{ Vaccine Strains } & \multicolumn{2}{|c|}{ Clinical Isolates } \\
\hline & $J 445$ & $\mathrm{~J} 446$ & $J 447$ & $J 448$ & BPD1 & BPD2 \\
\hline Tohama-I & 98.7038 & 98.6752 & 97.1793 & 95.6999 & 97.8997 & 98.5198 \\
\hline$J 445$ & - & 98.908 & 98.3484 & 96.8588 & 98.9038 & 99.69 \\
\hline$J 446$ & 98.908 & - & 97.3909 & 95.9258 & 98.0163 & 98.7242 \\
\hline$J 447$ & 98.3484 & 97.3909 & - & 98.5076 & 97.4165 & 98.0679 \\
\hline$J 448$ & 96.8588 & 95.9258 & 98.5076 & - & 95.9406 & 96.5784 \\
\hline BPD1 & 98.9038 & 98.0163 & 97.4165 & 95.9406 & - & 99.1836 \\
\hline BPD2 & 99.69 & 98.7242 & 98.0679 & 96.5784 & 99.1836 & - \\
\hline
\end{tabular}

Column 1 in table represents the comparator against which the strains (highlighted in rows) were compared data suggests that the pan-genome of strains appears open but slowly closing.

To estimate a general functional role of the CDS present in the average genome of eight strains used in this study, the clusters of orthologous groups of proteins (COGs) for each of the genome were determined. The top four COG categories observed in all eight genomes were designated as, I (Lipid transport and metabolism, E (Amino acid transport and metabolism), K (Transcription) and $\mathrm{P}$ (Inorganic ion transport) while the lowest category containing COG genes were F (Nucleotide transport and metabolism), D (Cell cycle control, cell division, chromosome partitioning) and $\mathrm{N}$ (cell motility) (Fig. 3). A total 23 functional categories were defined in the Tohama-I strain according to COG analysis [32]. In comparison with the reference strain, only 20 functional categories were observed for eight strains used in this study. The categories absent in vaccine and clinical genomes as compared to Tohama-I were related to genes involved in nucleotide metabolism, membrane transport and iron metabolism.

\section{Mobilome analysis}

Mobilome or mobile genetic elements (MGEs) include insertion sequences, bacteriophages, and genomic islands (GIs). B. pertussis genome has more than 200 copies of insertion sequence (IS elements) [33, 34]. ISs present in 5 vaccine and 2 clinical strains mainly belonged to three IS families IS481, IS1002, IS1663 (Additional file 2). The average copy number observed for IS481, IS1001 and IS1002 of genomes used in this study was 257, 20 and 7, respectively as discussed earlier (Table 1). We observed a slight increase in the copy number of IS481 similar with reports from countries using ACV.

PHASTER tool was used to identify phage region in all genomes in this study. Potential prophage sequences in the genome were identified and categorized as intact, incomplete or questionable [59]. Only in clinical isolate $\mathrm{BPD} 2$, we observed one intact phage region called as phage 1 which consists of $20.3 \mathrm{~kb}(1627048-1,647,419 \mathrm{bp})$ region having $62.30 \% \mathrm{G}+\mathrm{C}$ content and a total of 24 CDS. Phage 1 region from BPD2 were typically found to contain several phage-associated genes (Additional file 2). Clinical isolate BPD1 also showed the presence of 5 regions, but none was intact ( 4 incomplete and 1 uncharacterized region) (Additional file 2). Vaccine strain J445, J446, J448 and other B. pertussis strains carried phage region similar to phage 1 . We also observed the presence of a similar region in other $B$. pertussis complete genomes available in databases by using BLAST analysis. Approximately 18\% (100 out of 551) of available complete B. pertussis genomes showed high similarity with phage 1 . We 


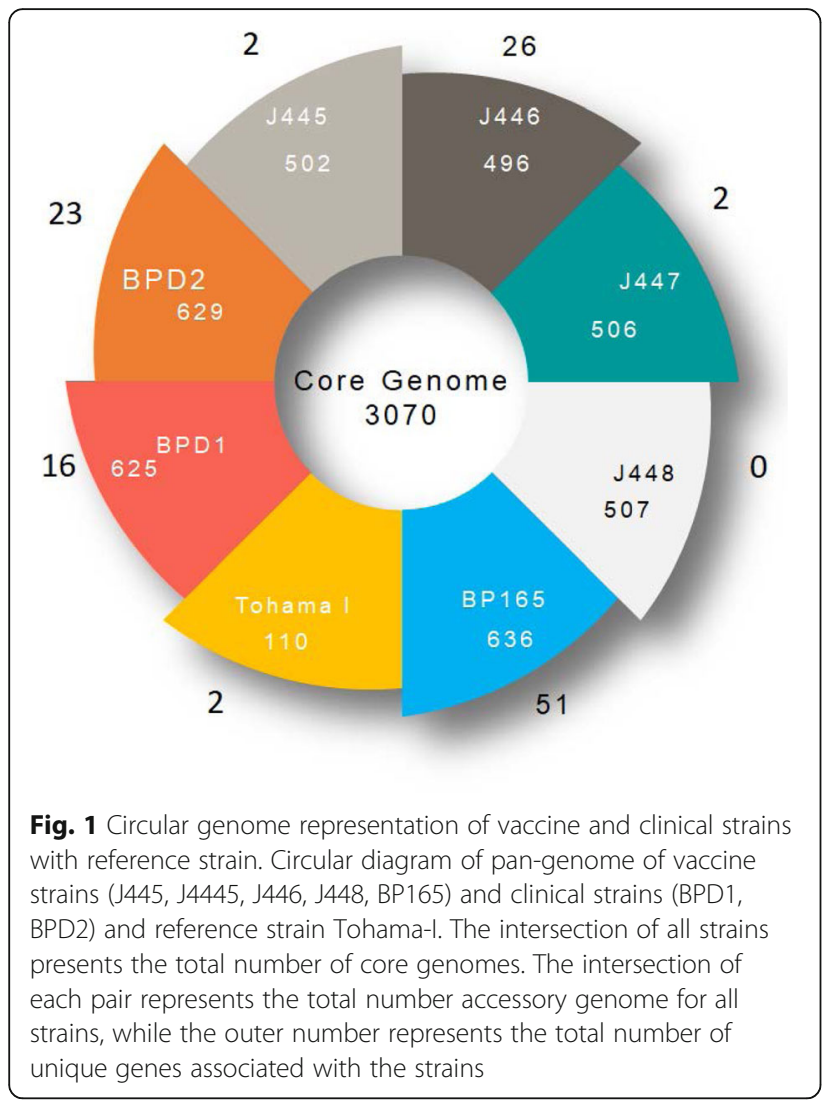

did not observe CRISPR sequences, plasmids and antibiotic resistance genes in any of the genomes.

The mutation associated with antibiotic (macrolide) resistance observed were from A to $G$ at position 2047 (A2047G) located in domain $\mathrm{V}$ of the 23S rRNA gene [63]. The reported A2047G position was based on old Tohama-I $23 \mathrm{~S}$ rRNA sequence, and it is equivalent to A2037G in the updated Tohama-I genome (Accession No. NC_002929.2). The 23S rRNA gene sequence of clinical isolates BPD1 and BPD2 were compared with 23S rRNA gene Tohama-I (X68323) and Chinese vaccine strain (CP002695), as all the PCR based diagnostic tools to detect the antimicrobial resistance mechanism in B. pertussis strains were based on these reference genomes. Based on whole genome sequence data we did not observe such mutation in clinical isolates BPD1 and BPD2 reported from India (Fig. 4).

Genomic plasticity reported in B. pertussis is through gene acquisition, gene loss and genomic organization [64]. Horizontal gene transfer (HGT) is also one of the mechanisms responsible for genome evolution. Genomic islands (GIs) are genomic fragments acquired by HGT events and may have an impact on the genome plasticity. We observed 31 GIs in BPD1 and BPD2 strains consisting of 484 and 528 genes, respectively (Additional file 3). Most of these genes were involved in carbohydrate, amino acid metabolism, membrane transport and transposases.

\section{cgMLST and phylogenetic analysis}

Genome sequences of vaccine and clinical strains were analyzed using the gene-by-gene approach known as

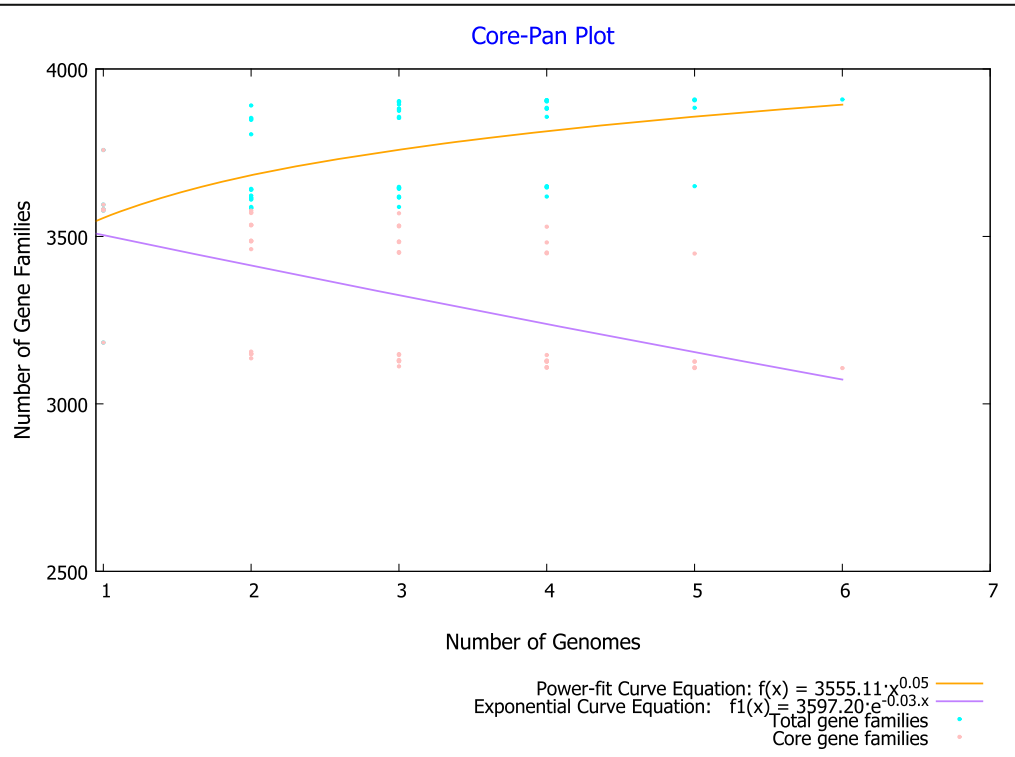

Fig. 2 Pan and core genome plot. Pan Genome and core genome plot of eight B. pertussis vaccine strains (J445, J4445, J446, J448, BP165) and clinical strains (BPD1, BPD2) and reference strain Tohama-l. The plot shows that progression of the pan (orange) and core (purple) genomes. The number of shared genes was plotted as the function of the number of strains (n) added sequentially with 3070 genes which were shared by genomes. The orange line represents the least-squares fit to the power-law function $f(x)=a \cdot x \wedge b$ where $a=3538.18, b=0.0289134$. The red line represents the least-squares fit to the exponential decay function $f 1(x)=c^{\wedge} e^{\wedge}(d . x)$ where $c=3672.32, d=-0.0317036$ 


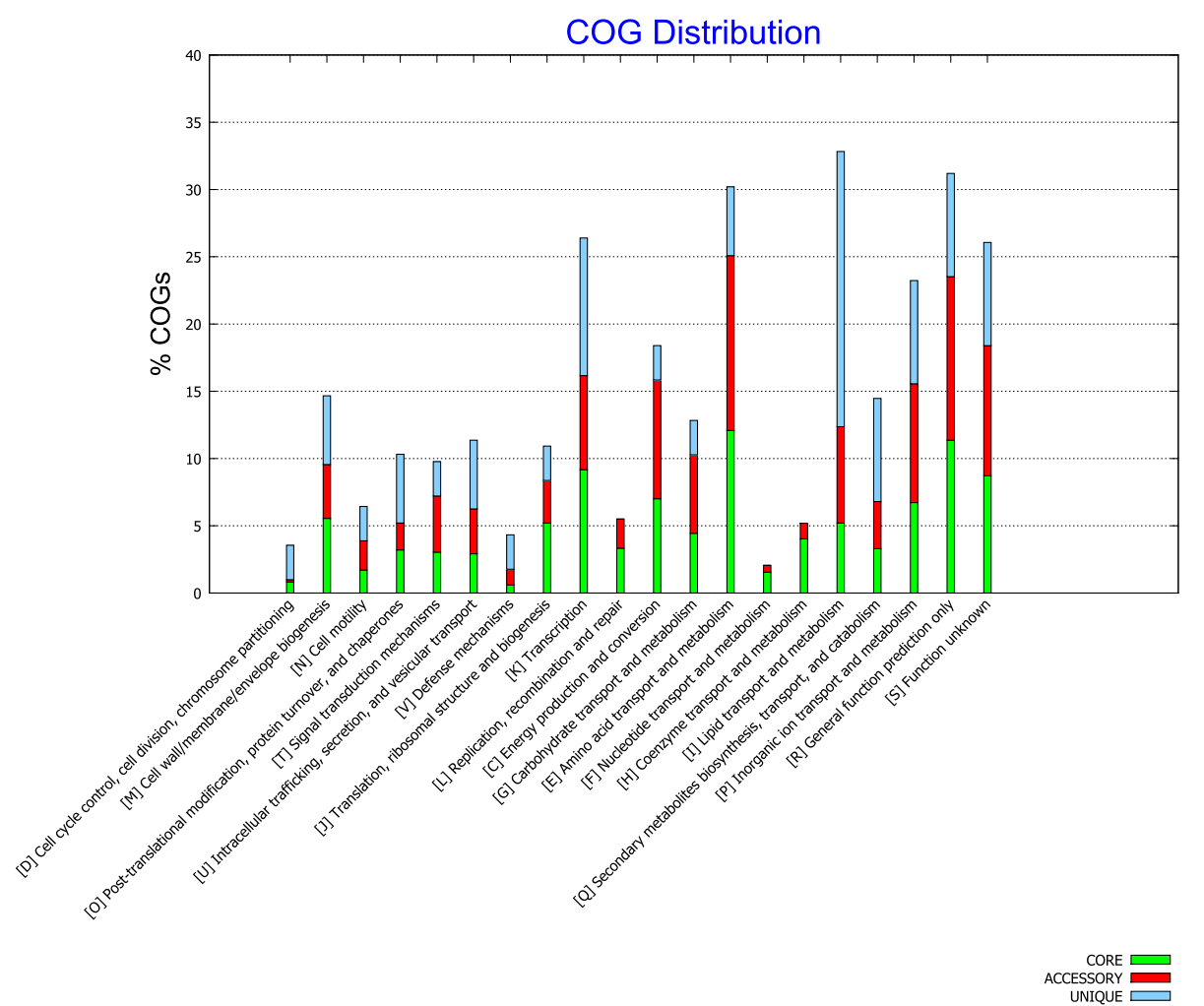

Fig. 3 Functional annotation with Clusters of Orthologous Genes (COGs). Functional annotation with Clusters of Orthologous Genes (COGs) assigned pan-genome of vaccine strains (J445, J4445, J446, J448, BP165) and clinical strains (BPD1, BPD2) and reference strain Tohama-I. The height of each bar represents a percentage of the core, accessory and unique genes involved in specific functional categories represented at the horizontal axis

core genome MLST (cgMLST). Recently cgMLST genotyping strategies were implemented for international coordinated surveillance of several pathogenic bacterial species. cgMLST has been recently developed for B. pertussis surveillance [45]. cgMLST scheme provides an excellent approach that combines high resolution of genome-level variation with high reproducibility. We compared vaccine and clinical strain genome sequences with the Bordetella spp. database (https://bigsdb.pasteur. $\mathrm{fr} /$ bordetella/). We recorded individual strain matching profiles, cgST profiles and number of mismatches with predefined 2038 core gene loci for each genome. Global comparison of Indian clinical B. pertussis isolates BPD1 and BPD2 with cgMLST database revealed 97.2\% (1981/ $2038)$ and $94.9 \%(1935 / 2013)$ similarity, respectively. Among the 2038 loci of the cgMLST scheme, 57 (BPD1)

\begin{tabular}{|c|c|}
\hline & \\
\hline pertussis CS CP002695.1 & \\
\hline Issis CS CPO02695.1 & . CAGTG. . C. . C \\
\hline ussis CS CP002695.1 & . CAGTG. . . . C \\
\hline I5sis BPD1 CP034182.1 & \\
\hline ssis BPD1 CP034182.1 & $\cdots$ \\
\hline 2.1 & $\cdots$ \\
\hline & . \\
\hline & \\
\hline $\mathrm{PD} 2 \mathrm{CPO} 34$ & \\
\hline
\end{tabular}

Fig. 4 Identification of single nucleotide polymorphism associated with antibiotic resistance using multiple genome alignment. Multiple sequence alignment generated using MEGA 9, with sequence of the $23 \mathrm{~S} \mathrm{rRNA}$ gene of Bordetella pertussis as reference sequence, showing sequence similarity of clinical strains BPD1 and BPD2 from India with 23S rRNA gene of Bordetella pertussis of Tohama-I and Chinese vaccine strain at position A2047G based on old Tohama-I 23 S rRNA gene of Tohama-l. Absence of nucleotide variation observed at position describing mutation associated with antibiotic resistance (binding site of erythromycin) 
and 103 (BPD2) loci showed differences with cgMLST database (Additional file 4). The cgST profiles were found to be similar for both clinical isolates (Table 3).

Phylogenetic analysis of 5 vaccines, 2 clinical isolates reported from India and reference strain was compared with 166 isolates from countries using ACVs. These isolates represent regions corresponding to France, US and UK [45]. Of these 166 isolates, 55 isolates from France corresponded to groups of intrafamilial or of multiple isolates from the same patient and randomly selected cocirculating isolates. Out of the remaining 111 isolates, corresponding to 3 outbreaks of pertussis that observed in ACV using countries like US and UK [57]. Core genome phylogenetic tree was constructed using amino acid sequences from cgMLST loci extracted for 166 B. pertussis isolates [45]. Phylogenetic tree was constructed with Bordetella parapertussis as an outgroup (Fig. 5). Phylogenetic analysis using 2038 core gene sequences of four Indian B. pertussis vaccine strains (J445, J446, J447 and J448) showed close genetic relatedness with Indian clinical isolates and Tohama-I (bootstrap 80). Vaccine strain J445, J446 formed a separate sub-cluster with Tohama-I (bootstrap 99) and strain J447 and J448 shared separate sub-cluster with isolates BPD1, BPD2 (Fig. 5). Interestingly, isolates H3755, 2,250,905, ERS227757 and FR6022 were found to be closely related with Indian vaccine strains and clinical isolates (bootstrap 80). Isolates H3755, 2,250,905 from US (California), ERS227757 from UK and FR6022 from France also shared closeness with Tohama-I. The closeness was further consistent with allelic profiles as isolates showed similar genetic profiles as $p \operatorname{txP} 1$, ptxA1, fim 2-1, fim3-1 with Tohama-I [45]. Out of all the vaccine strains, BP165 was found to be distant from Indian clinical isolates. This could be attributed to the origin of $\mathrm{BP}$ 165 , as it is a US isolate. BP165 clustered closely with isolates ERS227758 reported from UK with a similar fim2-1, prn1 allele profile. BP165 was also found to form a separate sub-cluster with isolate ERS227764 having PtxP3 allele.

\section{MLST and genotyping}

Bordetella MLST database classifies Bordetella genus into 43 sequence types (STs) and 4 clonal complexes
(CCs). Of these CCs, CC2 belongs to B. pertussis and is composed of 3 sequence types, ST1, ST2, and ST24 [1, 65]. Sequence type (ST-2) reportedly covers most of the circulating strains and is a dominant sequence type since late 1990s [65]. ST1 profile represents largely ancient strains such as Tohama-I [61, 62]. MLST analysis suggests that four vaccine strains (J445, J447, J448 and BP165) and two clinical isolates (BPD1 and BPD2) belong to ST-2 class (Table 3). Whereas, J446 strain showed ST-1 profile.

Globally, B. pertussis isolates are characterized based on allelic profiles of major virulence genes including promoter sequence of pertussis toxin gene (ptxP), pertussis toxin (ptxA), pertactin (prn) and fimbriae (fim2 and fim3) [66, 67]. These virulence-associated genes have shown divergence from vaccine reference strains [16-19]. Vaccine and clinical isolates were subjected to allelic profiling. Both Indian clinical isolates showed allele profiles as (BPD1:ptxP1/ptxA1/prn1/fim2-1 and BPD2: ptxP1/ptxA1/prn1/fim2-1) which were found similar to WCV vaccine strains (J445:ptxP1/ptxA2/prn1/ fim2-1/fim3-1; J446: ptxP2/ptxA4/prn7/fim2-2/fim3-1; J447 and J448: ptxP1/ptxA1/ prn1/fim2-1/fim3-1). We also studied allelic profiles of other virulence-associated genes such as tracheal colonization factor (tcf-A), Bordetella associated protein $\mathrm{C}$ (BapC), Adenylate cyclase (cyaA), outer membrane protein $\mathrm{Q}$ and Virulence associated gene (Vag8) in vaccine and clinical isolates. The Indian clinical isolates and vaccine strains demonstrated similar profiles as (tcfA-2-tcfA-9, bapC1, cyaA2, ompQ1ompQ2, vag8).

\section{Gene loss and duplication}

Number of genes lost or duplicated in clinical and vaccine strains was studied as compared to reference strain Tohama-I. The study suggests that vaccine strains and clinical isolates displayed gene loss and duplication with no significant impact on overall genome size as the number of gene lost was nearly equal to the number of genes duplicated (Table 4). Genomic deletions and ongoing gene loss are one of the apparent features observed in recent clinical isolates

Table 3 MLST and cgMLST profiles of strains

\begin{tabular}{lllllllllll}
\hline Feature & Strain & adk & FumC & glyA & TyrB & Icd & pepA & Pgm & ST & cg-MLST \\
\hline Vaccine strains & J445 & 1 & 1 & 1 & 3 & 1 & 1 & 1 & 2 & 413 \\
& J446 & 1 & 1 & 1 & 1 & 1 & 1 & 1 & 1 & 410 \\
& J447 & 1 & 1 & 1 & 3 & 1 & 1 & 1 & 2 & 411 \\
& J448 & 1 & 1 & 1 & 3 & 1 & 1 & 1 & 2 & 412 \\
& BP 165 & 1 & 1 & 1 & 3 & 1 & 1 & 1 & 2 & 41 \\
& BPD1 & 1 & 1 & 1 & 3 & 1 & 1 & 1 & 2 & 362 \\
& BPD2 & 1 & 1 & 1 & 3 & 1 & 1 & 1 & 2 & 362 \\
\hline
\end{tabular}




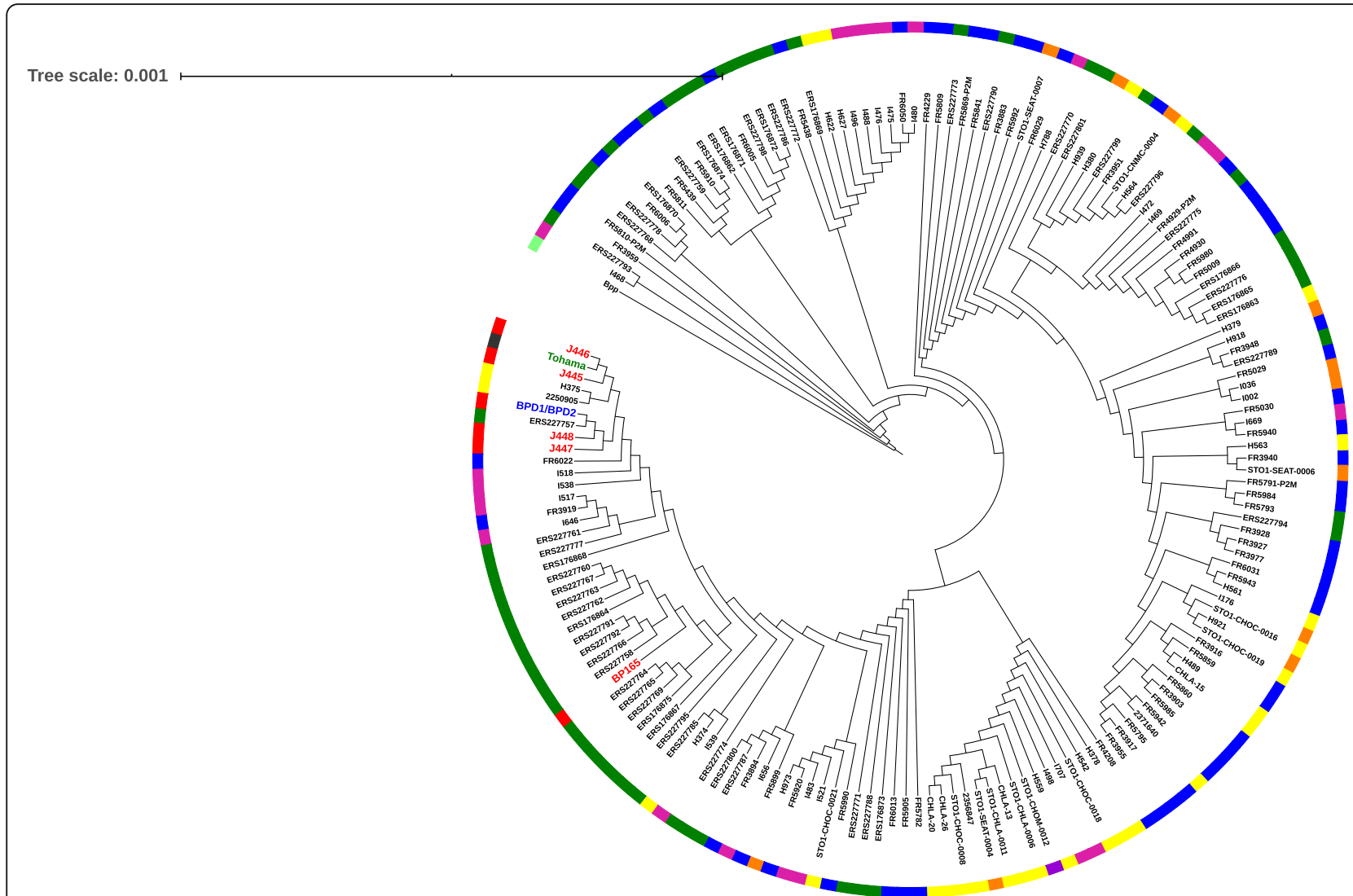

Fig. 5 Phylogenetic analysis of vaccine and clinical strains using cgMLST. Maximum-likelihood phylogenetic tree for Indian Bordetella pertussis isolates and vaccine strains and isolates from other geographical origins based on concatenated alignments of 2038 cgMLST gene sequences. The tree was rooted with strain Bordetella parapertussis (GenBank Accession number CP019931.1). Tree scale is indicated above the diagram. The external circle indicates the geographical origin of isolates (blue, France; red, India; Pink, Vermont; Green, UK; Yellow, California; Orange, Washington; Purple, Michigan). Vaccine strains and Indian Isolates labels highlighted in bold red colour. The scale bar indicated amino-acid substitution per site. The tree was constructed with bootstrap values 1000 replicates. Bootstrap values are indicated with the position on branch 50 and scale by factor 1

reported from many countries $[44,55]$. The lost genes majorly belonged to categories of membrane transport and amino acid metabolism. The genes identified in the category of amino acid metabolism were related to $\mathrm{ABC}$ transporter's components. $\mathrm{ABC}$ transporters are a large group of proteins which have cellular functions in import and export various substances, which suggests deletion of such genes might confer a selective advantage as a one of the strategies during adaptation of the organism in highly vaccinated populations $[44,48,68]$. The duplicated genes in all strains found in COG categories belonged to amino acid and nucleic acid metabolism and post-translation modification (Additional file 5). We did not observe any gene loss or gene acquisition of virulence-associated genes.

Table 4 Gene loss and duplication in strains

\begin{tabular}{llllll}
\hline Feature & Strains & No of absent genes & No of gain genes & No of duplicated genes & No of genes defined in COG (\%) \\
\hline Vaccine Strains & J445 & 393 & 36 & 388 & - \\
& J446 & 393 & 37 & 387 & 18 \\
& J447 & 393 & 36 & 448 & - \\
Clinical strains & J448 & 394 & 36 & 446 & - \\
& BPD1 & 401 & 36 & 401 & - \\
Reference strain & BPD2 & 395 & 36 & 388 & 387 \\
\hline
\end{tabular}




\section{Whole genomic comparison}

A BLAST Atlas comparing all seven genomes with reference genome was generated using the Gview comparison tool with e value $1 \mathrm{e}-10$ and percent identity cutoff (removing matches below percent identity) of $80 \%$. A circular genome map with each genome represented by the ring was constructed with center circle representing Tohama-I. A visual inspection of the circular alignment of the genomes revealed a high sequence similarity in all genomes with reference genome; especially in the region 1 (approximately1-850 bp and the region of 3250-3780 bp) (Fig. 6). This region was identical in all strains with $100 \%$ identity (Additional file 6). Also, region 2 (1500$3000 \mathrm{bp}$ ) showed more than $99 \%$ identity. We found a small gap (region 3) in strains J445, J447, J448, BPD1 and BPD2 between 900 and 1000 bp (Fig. 6). The genes observed in this gap were related to amino acid and nucleotide metabolism. We also observed a small gap in region 4 (1300-1500 bp) in clinical strains as compared to vaccine strains. The circular genome map comparison showed that all strains especially clinical strains are identical with vaccine and reference strain, with slight gene loss detected. All the absent genes in clinical isolates were involved in amino and nucleotide metabolism.

Genome structure has important effects on bacterial phenotypes and the evolution of bacterial genomes. To detect overall chromosomal rearrangements, deletions, duplications among vaccine strains, and clinical strains with the reference genome, we aligned the genomes using Mauve v2.0 [82]. The multiple whole-genome alignment was conducted using the progressive alignment algorithm implemented in Mauve v2.0 using default parameters. Alignment output generated 5,064,183 root alignment length and 103 super intervals. Mauve

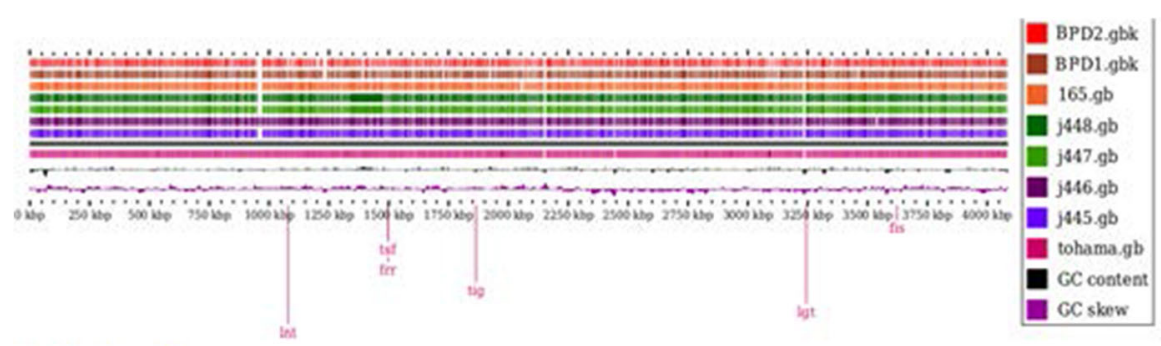

A
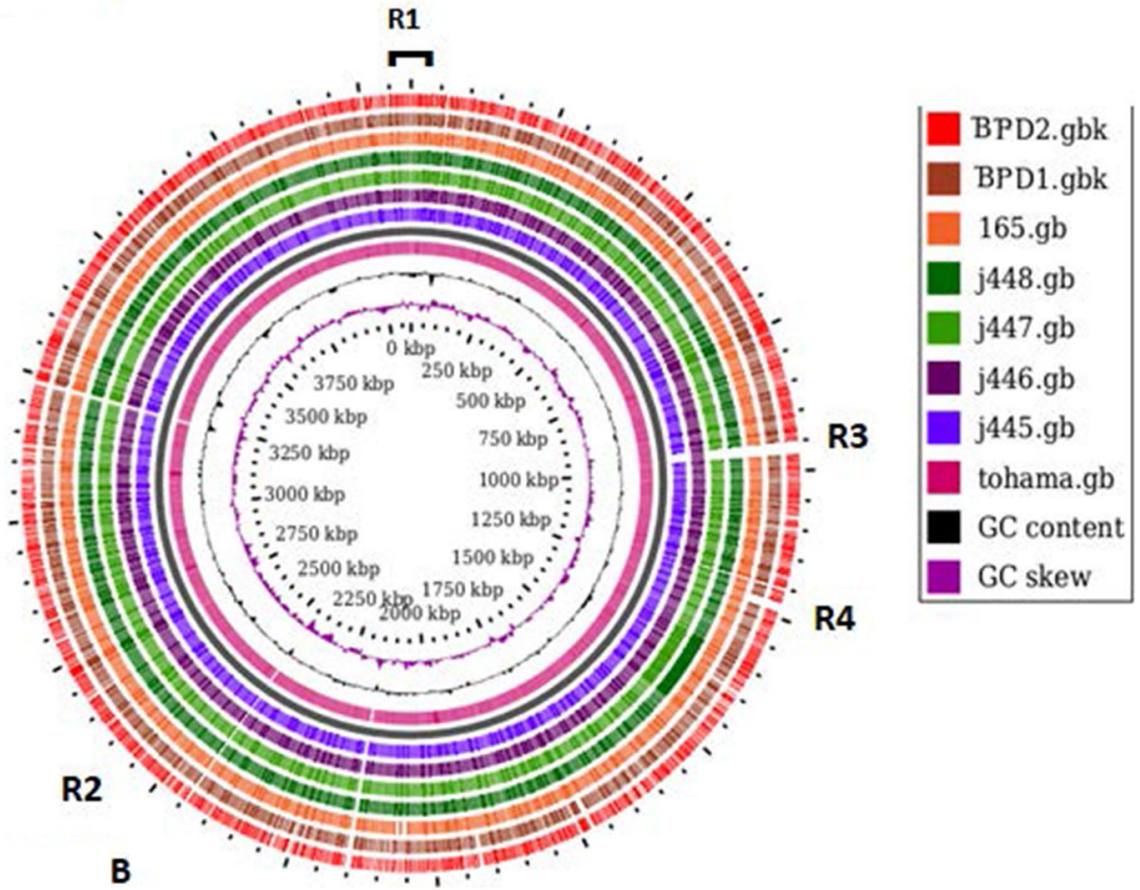

Fig. 6 BLAST Atlas comparing eight B. pertussis strains. BLAST Atlas comparing eight B. pertussis vaccine strains (J445, J4445, J446, J448, BP165) and clinical strains (BPD1, BPD2) against reference strain Tohama-l. a. The linear arrangement of genomes of the vaccine strain, clinical strain genomes similarity with reference strain Tohama-I. b. Circular plot generated using G view Comparison Tool using BLASTn. Genomes are arranged with vaccine strains and clinical strains from innermost ring close to the reference genome to outermost ring from reference genome: inner circle reference strain Tohama-I (pink) GC content (black), G + C skew (purple) 
showed a total of 56 localized collinear blocks (LCB) in the analyzed genomes, which suggest the presence of rearrangement observed in vaccine and clinical genomes as compared to reference genome (Additional file 7). An LCB represents a conserved region within genomes with exactly matched sequences that are shared by genomes aligned. Some LCBs are too small to display on the figure (Fig. 7). LCB boundaries were found using two programs, project And Strip, and makeBadgerMatrix, to generate LCB boundary files from the Mauve alignment.

Mauve detected a total of 20 inversions observed in clinical isolates BPD1 and BPD2 with reference Tohama-I, where these 20 blocks varied in size (Additional file 7). The major inversion is seen in BPD1 (located at 2142133-2379723) with LCB length 237,590 and in BPD2 (located at 3172139-3420774) with LCB length 248,635 was detected. Few small-scale inversions were also observed (Additional file 7). Few chromosomal arrangements like translocations were also found within BPD1 and BPD2 when compared to reference strain and vaccine strains.

\section{Discussion}

Antigenic divergence among circulating strains of B. pertussis is reported globally [69-73]. The impact of WCV on the circulating strains needs to be established. Manufacturing of WCV involves the use of more than one strain. The strains used in manufacturing vary globally. Therefore, genetic characterization of vaccine strains is important. Whole genome based comparative genomics offers high resolution data to study sequence and structural related variations. Till recently, such data was available for vaccine and circulating strains from countries using $\mathrm{ACV}$. We report here comparative genome analysis of vaccine strains with the reference strain, clinical isolates reported from India and isolates from countries using ACV.

The study includes analysis of genome characteristics, genomic structure and rearrangement, mobilome analysis, phylogeny, virulence factor typing, MLST and cgMLST analysis of pertussis strains. Comparative whole genome analysis of clinical isolates reported from India displayed $\geq 95 \%$ genome similarity with reference and WCV strains used in India. Further, mobilome analysis of clinical isolates from India suggests slight variation in number of pseudogenes, ISs among the strains. These differences in pseudogenes could be ascribed to the fact that vaccine strains are stored frozen in optimized conditions and have limited exposure to host. IS elements especially IS481 in B. pertussis strains is reported to vary among circulating strains [55-57]. Increased copy numbers of IS481 were also observed in Indian clinical isolates. A higher number of IS elements in monomorphic populations such as $B$. pertussis are known to provide

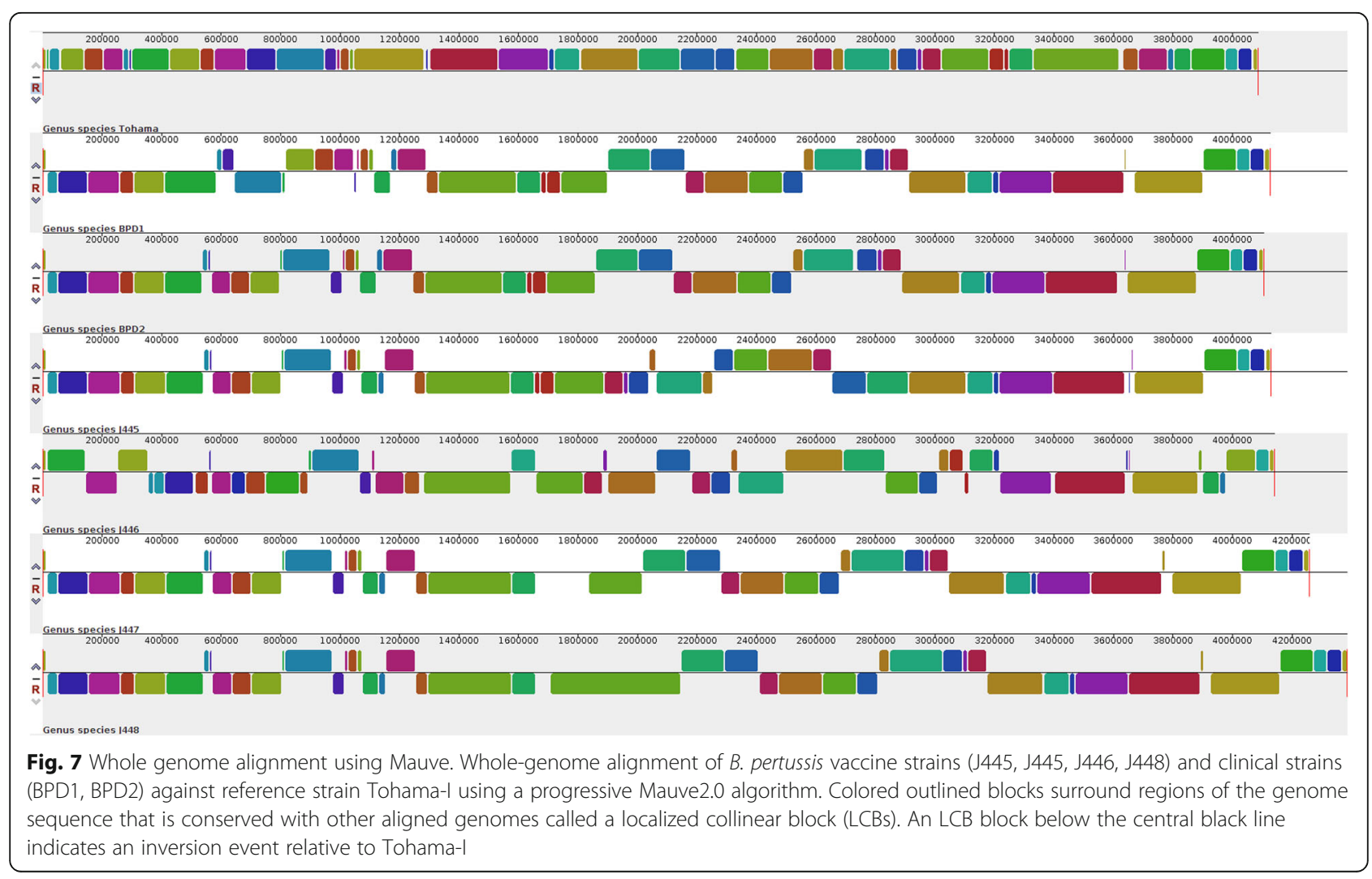


competitive advantage because of its role in genome reduction and genomic rearrangements [53, 54]. Bacteriophages contribute actively to bacterial evolution by integrating and excising from the genome [58]. In certain conditions, they provide new genetic properties to the bacterial host and leading to the development of new virulence within species. Indian clinical isolates and WCV strains showed the absence of phages, CRISPR and plasmids amongst the genomes which is significant towards the minimal genome plasticity. IS elements among the strains J445, J446, J447, J448, BP165 and BPD1 did not showed association with any virulenceassociated genes and its controlling elements. Overall, mobilome analysis observations are significant towards the stability of vaccine and clinical genomes.

Antibiotic treatment and prophylaxis constitute an essential part of interventions used to treat bacterial diseases. Macrolides including azithromycin, clarithromycin, and erythromycin remain the mainstay of treatment for pertussis [60]. Few reports have demonstrated antibiotic resistance against antibiotics like macrolide, erythromycin (minimum inhibitory concentration MIC $>256 \mathrm{IU} / \mathrm{L}$ ) in circulating strains of B. pertussis [61, 62]. Antibiotic resistance plays a key role in the resurgence of infectious disease. First erythromycin resistance cases were reported from the United States in 1994 [85]. Resistant strains were also reported from countries like France and China [61, 62]. In a recent study, antibiotic resistant strains were reported from Iran which is using WCV in its immunization programme [86]. This led us to monitor the emergence of antibiotic resistance in strains reported from India. Absence of antibiotic resistance genes observations in Indian clinical isolates need confirmatory studies with pan India isolates. Such data will be further useful to decipher how antibiotic resistance evolves in countries which are using WCVs as selection pressure may be different from what we have observed in countries which are using ACV.

Clonal expansion of $B$. pertussis circulating strains is reported in countries using ACV [28]. However, there is scarcity of data available from countries using WCV. Core genome based phylogenetic analysis of WCV strains showed close evolutionary relationship with reference Tohama-I strain and Indian clinical isolates suggesting lesser genetic diversity in Indian strains. Indian clinical isolates as compared to isolates reported from $\mathrm{ACV}$ were found to cluster distinctly, indicating minimal intermixing from global strains. Use of WCV in primary immunization programs can be one possible reason for lesser diversity in Indian strains. MLST and genotyping analysis was carried out for WCV strains. Out of 4 strains of WCV, 3 strains were found to have ST-2 profile, where 1 strain showed ST-1 and allele profile of virulence associated genes was similar in vaccine and clinical strains. Thus, a vaccine manufactured using combination of such strains may allow better coverage against circulating strains. However, this needs confirmation with a greater number of isolates from India and other WCV using countries.

Our initial findings do support the emerging hypothesis that B.pertussis evolution is associated with vaccine usage and this evolution is moving at different rates in different countries. However, it needs validation using a larger collection of clinical isolates from countries using WCV like India. Nevertheless, this study provides necessary data on WCV strains to study the evolution and diversity of B.pertussis in developing countries.

\section{Conclusions}

The study provides a comprehensive genomic analysis of WCV strains and clinical isolates from India which will be useful in facilitating surveillance of pertussis in India and its comparisons with globally reported trends in $B$. pertussis populations.

\section{Methods \\ Strain selection}

A total 5 vaccine (J445, J446, J447, J448, BP165), 2 clinical (BPD1, BPD2) and 1 reference strain (Tohama-I) were used in this study. Four strains (J445, J446, J447, and J448) were used in manufacturing of WCVs [42]. Strain J445 and J446 were procured from the culture collection of the Rijks Institute voor de Volksgezondheid, Netherlands. Strain J447 and J448 were obtained from the Lister Institute in London, England. Strain BP165 is a US clinical isolate procured from the Center for Biologics Evaluation and Research (CBER, United States) and used in manufacturing of acellular vaccines [43]. Strain BPD1 and BPD2 were clinical strains reported from India were used. Tohama-I was used as reference genome. Accession number of all the strains is provided in Table 1. Genomic sequences of 166 isolates used in development core genome MLST database of $B$. pertussis strains were used in this study. These isolates represent region correspond to France, US and UK [45]. Of these 166 isolates, 55 isolates from France corresponded to groups of intrafamilial or of multiple isolates from the same patient and randomly selected cocirculating isolates. Out of the remaining 109 isolates, 55 isolates corresponding to 2 outbreaks of pertussis that observed in ACV using countries like US and 54 isolates from UK.

\section{Genome sequencing and annotation}

Genomes of all strains were compared with reference strain Tohama-I. J445, J446, J447, J448 vaccine strains were sequenced using a combination of the PacBio RSII and Illumina MiSeq platforms as described previously [42], and genome sequences were deposited in GenBank 
databases under accession numbers available in Table 1 [38]. Genomic DNA extraction of fifth vaccine strain BP165 was performed using the Genomic DNA Clean and Concentrator - 10 Kit (ZYMO Research) as per the manufacturer's instructions, and DNA was quantified using nanodrop and Qubit. Sequencing was performed by preparing shotgun libraries using NEB Next Ultra DNA library prep kit. Sequencing was performed using platform Illumina MiSeq with 150 read data length (100X coverage) using paired-end sequencing. The whole-genome sequence of strain BP165 has been deposited in GenBank under the accession number RSFF00000000 [43]. One hundred and sixty-six isolates available from ACV countries available from databases were used for phylogenetic analysis [45]. Annotation of all the sequenced genomes used in this study (J445, J446, J447, J448, BP165 and BPD1, BPD2) was completed using automated annotation tool, to avoid the possible deviations due to different annotation methods [74].

\section{Pan-genome analysis}

Pan-genome analysis was performed using a bacterial pan-genome analysis (BPGA-ultra-fast bacteria) pipeline [50]. To depict the core and accessory genomes in each genus, a reciprocal best hit search using the BPGA software was performed. Pan-core plot against combinations will give core and pan-genome boxplot, and dot plot was generated using the desired number of unique combinations of genomes. Orthologous groups (OGs) of genes were observed using the orthoMCLv.2.0.9 software package (default parameters-value cutoff of 10-5, per cent match cutoff 50\%, MCL algorithm inflation value 1.5). Homologous clusters from OrthoMCL were compiled to show shared and unique genes [75].

\section{Mobilome analysis}

All strains were investigated for the presence of mobile genetic elements (MGEs) like bacteriophage, Insertion sequences, Genomic islands and Prophages. Potential prophage sequences in the genome were found and categorized (intact, incomplete or questionable) using PHASTER tool (http://phaster.ca/). A prediction of whether phage region has an intact or incomplete prophage was based on score value, which is for intact $(>90)$, questionable (70-90), and for incomplete $(<70)$ [59]. Insertion sequences located within the genome were identified using ISfinder (https://isfinder.biotoul.fr/) [76]. Additionally, each of the genomes was manually reviewed for the copy number of transposes sequences. Genomic islands (GIs) were predicted by using IslandViever3, including IslandPick, IslandPath-DIMOB, and SIGI-HMM [77]. Pseudogenes were predicted using NCBI Prokaryotic Genome Annotation Pipeline [78].
Virulence genes were identified using Virulence Finder, and Antibiotic resistance genes (ARGs) using a combination of ResFinder and the Comprehensive Antibiotic Resistance Database (CARDs) [79].CRISPER Finder (http://crispr.u-psud.fr/) was used to identify clustered regularly interspaced short palindromic repeats (CRISPRs) [80].

\section{Whole-genome comparison}

A BLAST Atlas comparing all genomes to the reference genome was generated by GView comparison tool [81]. Genome organization was studied by aligning sequenced genomes using a progressive Mauve algorithm (version 2.0) with default parameters [82]. All the tools used with the default settings.

\section{MLST and cg-MLST analysis}

MLST was performed using www.pubmlst.org(PubMLST) Bordetella spp. database based on housekeeping genes (adenylate kinase, fumarate hydratase class II, aromatic amino-acid aminotransferase, isocitrate dehydrogenase, cytosol aminopeptidase, and phosphoglucomutase) and genotyping of antigenic determinant genes (pertussis toxin subunit 1, pertactin, filamentous hemagglutinin, fimbriae2, fimbriae3, outer-membrane protein Q, Bordetella anti-transporter-protein C, adenylate cyclase toxin, virulence-activated gene 8 , and tracheal colonizing factor) was also studied. The allele of each gene and the sequence type (ST) were defined according to the public database [33, 46]. cgMLST was analyzed using a publicly available web-accessible genotyping platform (https://bigsdb.pasteur.fr/bordetella) [45]. Briefly, genome assemblies were compared using BLASTN to the reference alleles of 2038 predefined gene loci, as previously described [51]. We evaluated individual comparison of each strain allelic profiles and recorded the number of mismatches, and defined cgST profile for each isolate according to cgMLST scheme of 2038 core gene loci. To derive a phylogenetic tree based on cgMLST loci, we extracted and aligned the amino acid sequences of all loci with MAFFTv7, as previously described [83]. We used IQ-TREEv1.5.4 to infer a maximum likelihood phylogenetic tree based on alignment of the sequences from each 2038 cgMLST loci. We assessed branch support with bootstrap (1000 replicates). Phylogenetic tree annotation and visualization were performed using iTOL $[78,84]$. Duplicates and lost genes were predicted solely based on Rapid Annotation using Subsystem Technology (RAST) annotation pipeline. The complete list of genes annotated in all vaccine and clinical genomes were evaluated and compared with reference genome [87]. 


\section{Supplementary information}

Supplementary information accompanies this paper at https://doi.org/10. 1186/s12864-020-6724-8.

Additional file 1. Genome neighbor report analysis of vaccine (J445, J446, J447, J448) and Clinical (BPD1, BPD2) strains. NCBI genome neighbor report analysis of vaccine and clinical strains suggesting \% symmetric identity with total available genomes in NCBI databases.

Additional file 2. Mobile genetic elements (Insertion Sequences, Prophages) identified in the Bordetella vaccine and clinical strains. Insertion sequences identified in eight B. pertussis vaccine strains (J445, J4445, J446, J448, BP165) and clinical strains (BPD1, BPD2) and reference strain Tohama-I using IS Finder with describing details about blast matched values, e values and IS families. The second sheet listing phage regions identified in all strains. Details of phage region with its nature and sequence are described in the last sheet.

Additional file 3. Genomic island region observed for B. pertussis vaccine strains. List and details of all $\mathrm{Gl}$ regions observed with coded genes information for strains (J445, J4445, J446, J448, and BP165) and clinical strains (BPD1, BPD2). Table describing the list of genes observed in each genomic island with a total length of island region (bp), start and end region, a gene present in particular island, Gene start and gene end, and product expressed by a particular gene.

Additional file 4. cgMLST Analysis. Table describing allelic profile observed for total 2038 genes with locus of each gene, allele number, and length of the gene, contigs number and starts, and end position of each gene observed in respective strain.

Additional file 5. List of genes absent and duplicated in strains. List of duplicated genes observed in the vaccine (J445, J4445, J446, J448, and BP165) and clinical strains (BPD1, BPD2) as compared to the reference strain. Table describing genes duplicated in particular strain (Highlighted part) with contig number, start and end position of genes, total length, and gene product with subsystem annotation.

Additional file 6. BLAST ATLAS. List of genes identified during BLAST ATLAS genome comparison with reference genome Tohama-l.

Additional file 7. Permutation matrix file. Permutation matrix file generated from MAUVE progressive alignment containing information about total LCB blocks observed during alignment generated and their relative position in each genome.

\section{Abbreviations}

WCVs: Whole-cell vaccines; ACVs: Acellular vaccines; cg-MLST: core genome multilocus typing; MLST: Multi-locus sequence typing; COG: Cluster of orthologous genes; MGEs: mobile genetic elements; ISs: Insertion sequences; MGEs: Mobile genetic elements; ST: Sequence type; Gls: Genomic islands; ptx: Pertussis toxin; prn: Pertactin; fim: Fimbrae; FHA: Filamnetous hemaglutinin; tcf: Tracheal colonization factor; CDS: Coding sequences; NCBI: National centre for Biological Sciences; CRISPER: Clustered Regularly Interspaced Short Palindromic Repeats; HGT: Horizontal gnee transfer; BapC: Bordetella associated protein c; cyaA: Adenylate cyclase; vag8: Virulence associated gene 8; LCB: Local collinearized Bloack; PATRIC: Pathosystems Resource Integration Center; CC: Clonal complex; iTol: Interactive Tree of Life; RAST: Rapid Annotation using Subsystem Technology.

\section{Acknowledgements}

The authors are thankful to the Serum Institute of India, National Centre for Microbial Resources (NCMR) and Symbiosis International University (SIU) for providing all the facilities and the assistance for carrying out this study. The findings and conclusions in this report are those of authors and do not necessarily represent the official position of the company or institute.

\section{Authors' contributions}

SG, US, MG and DD conceived and designed the study. The analysis of the data was carried out by SA, VG, SP and KP. The manuscript was drafted by SA, VG and KP and reviewed by SG, US, MG and DD. All authors read, commented and approved the final manuscript.

\section{Funding}

This work was supported by the Serum Institute of India Pvt. Ltd., Pune (SIIPL) and Department of Health and Biological Science, Symbiosis International University, Pune (SIU), as a part of doctoral research program. Authors from SIIPL and SIU were involved in the design of the study and analysis, interpretation of data and writing the manuscript.

\section{Availability of data and materials}

The datasets generated or analyzed during the current study are available in the National Center for Biology Information (NCBI), GenBank repository, [under accession numbers as CP017402, CP017403, CP017404, CP017405, RSF00000000, CP034182, CP0134101]". Most other datasets obtained from web-based sources are included as weblink in "Method" section and "Additional files".

Ethics approval and consent to participate

Not Applicable.

\section{Consent for publication}

Not Applicable.

\section{Competing interests}

The authors declare that they have no competing interests.

\section{Author details}

${ }^{1}$ Department of Health and Biological Sciences, Symbiosis International University, Pune, Maharashtra 412115, India. ${ }^{2}$ National Centre for Microbial Resource, National Centre for Cell Science, Pune, Maharashtra 411021, India. ${ }^{3}$ Serum Institute of India Pvt. Ltd, Pune, Maharashtra 411028, India.

Received: 11 January 2020 Accepted: 6 April 2020

Published online: 07 May 2020

\section{References}

1. Diavatopoulos DA, Cummings CA, Schouls LM, Brinig MM, Relman DA, Mooi FR. Bordetella pertussis, the causative agent of whooping cough, evolved from a distinct, human-associated lineage of B. bronchiseptica. PLoS Pathogens. 2005;1(4):e45

2. Lambert LC. Pertussis vaccine trials in the 1990s. J Infect Dis. 2014; 209(suppl_1):S4-9.

3. Mooi FR. Bordetella pertussis and vaccination: the persistence of a genetically monomorphic pathogen. Infect Genet Evol. 2010;10(1):36-49.

4. Robinson A, Irons LI, Ashworth LA. Pertussis vaccine: present status and future prospects. Vaccine. 1985:3(1):11-22.

5. Domenech de Cellès M, Magpantay FM, King AA, Rohani P. The pertussis enigma: reconciling epidemiology, immunology and evolution. Proc Biol Sci. 2016;283(1822):20152309

6. Tan T, Dalby T, Forsyth $K$, Halperin SA, Heininger U, Hozbor D, Plotkin S, Ulloa-Gutierrez R, Von König CH. Pertussis across the globe: recent epidemiologic trends from 2000 to 2013. Pediatr Infect Dis J. 2015;34(9): e222-32

7. Jackson DW, Rohani P. Perplexities of pertussis: recent global epidemiological trends and their potential causes. Epidemiol Infect. 2014; 142(4):672-84

8. Cherry JD. Epidemic pertussis in 2012 - the resurgence of a vaccinepreventable disease. N Engl J Med. 2012;367(9):785-7.

9. Rohani P, Drake JM. The decline and resurgence of pertussis in the US. Epidemics. 2011;3(3-4):183-8.

10. Moerman L, Leventhal A, Slater PE, Anis E, Yishai R, Marva E. The reemergence of pertussis in Israel. Isr Med Assoc J. 2006;8(5):308-11.

11. Scheil W, Cameron S, Roberts C, Hall R. Pertussis in South Australia 1893 to 1996. Commun Dis Intell. 1998:22:76-9.

12. Quinn HE, Mclntyre PB. The impact of adolescent pertussis immunization, 2004-2009: lessons from Australia. Bull World Health Organ. 2011;89:666-74.

13. Mooi FR, Van Der Maas NA, De Melker HE. Pertussis resurgence: waning immunity and pathogen adaptation-two sides of the same coin. Epidemiol Infect. 2014;142(4):685-94.

14. He Q, Mertsola J. Factors contributing to pertussis resurgence. Future Med. 2008:329-339. 
15. Preston A. The role of B. pertussis vaccine antigen gene variants in pertussis resurgence and possible consequences for vaccine development. Hum Vaccin Immunother. 2016;12(5):1274-6.

16. Fry NK, Neal S, Harrison TG, Miller E, Matthews R, George RC. Genotypic variation in the Bordetella pertussis virulence factors pertactin and pertussis toxin in historical and recent clinical isolates in the United Kingdom. Infection and immunity. 2001;69(9):5520-8.

17. Bottero D, Gaillard ME, Basile LA, Fritz M, Hozbor DF. Genotypic and phenotypic characterization of Bordetella pertussis strains used in different vaccine formulations in Latin America. J Appl Microbiol. 2012;112(6):126676.

18. Yao SM, Lin YC, Chou CY, Chen YY, Hsiao MJ, Chen HY, Yan JJ, Su HP, Li SY. Antigenic divergence of Bordetella pertussis isolates in Taiwan. J Clin Microbiol. 2005;43(11):5457-61

19. Packard ER, Parton R, Coote JG, Fry NK. Sequence variation and conservation in virulence-related genes of Bordetella pertussis isolates from the UK. J Med Microbiol. 2004:53(5):355-65.

20. Bouchez V, Hegerle N, Strati F, Njamkepo E, Guiso N. New data on vaccine antigen deficient Bordetella pertussis isolates. Vaccines. 2015;3(3):751-70.

21. Souder E, Long SS. Pertussis in the era of new strains of Bordetella pertussis. Infect Dis Clin. 2015;29(4):699-713.

22. Zeddeman A, Gent MV, Heuvelman C, Heide H, Bart M, Advani A, Hallander $\mathrm{H}$, Wirsing von Konig C, Riffelman M, Storsaeter J, Vestrheim D. Investigations into the emergence of pertactin-deficient Bordetella pertussis isolates in six European countries, 1996 to 2012. Euro Surveill. 2014;19(33): 20881.

23. Bouchez V, Brun D, Cantinelli T, Dore G, Njamkepo E, Guiso N. First report and detailed characterization of B. pertussis isolates not expressing pertussis toxin or pertactin. Vaccine. 2009;27(43):6034-41.

24. Pawloski LC, Queenan AM, Cassiday PK, Lynch AS, Harrison MJ, Shang W, Williams MM, Bowden KE, Burgos-Rivera B, Qin X, Messonnier N. Prevalence and molecular characterization of pertactin-deficient Bordetella pertussis in the United States. Clin Vaccine Immunol. 2014;21(2):119-25.

25. Queenan AM, Cassiday PK, Evangelista A. Pertactin-negative variants of Bordetella pertussis in the United States. N Engl J Med. 2013;368(6):583-4.

26. Dorji D, Mooi F, Yantorno O, Deora R, Graham RM, Mukkur TK Bordetella pertussis virulence factors in the continuing evolution of whooping cough vaccines for improved performance. Med Microbiol Immunol. 2018;207(1):3-26.

27. Van Gent M, Pierard D, Lauwers S, Van Der Heide HG, King AJ, Mooi FR. Characterization of Bordetella pertussis clinical isolates that do not express the tracheal colonization factor. FEMS Immunol Med Microbiol. 2007;51(1): 149-54.

28. Bart MJ, Harris SR, Advani A, Arakawa Y, Bottero D, Bouchez V, Cassiday PK, Chiang CS, Dalby T, Fry NK, Gaillard ME. Global population structure and evolution of Bordetella pertussis and their relationship with vaccination. mBio. 2014;5(2):e01074-14.

29. Marchand-Austin A, Tsang RS, Guthrie JL, Ma JH, Lim GH, Crowcroft NS, Deeks SL, Farrell DJ, Jamieson FB. Short-read whole-genome sequencing for laboratory-based surveillance of Bordetella pertussis. J Clin Microbiol. 2017; 55(5):1446-53.

30. Xu Y, Liu B, Gröndahl-Yli-Hannuksila K, Tan Y, Feng L, Kallonen T, Wang L, Peng D, He Q, Wang L, Zhang S. Whole-genome sequencing reveals the effect of vaccination on the evolution of Bordetella pertussis. Sci Rep. 2015; 5:12888.

31. Maharjan RP, Gu C, Reeves PR, Sintchenko V, Gilbert GL, Lan R. Genome-wide analysis of single nucleotide polymorphisms in Bordetella pertussis using comparative genomic sequencing. Res Microbiol. 2008; 159(9-10):602-8.

32. Heikkinen E, Kallonen T, Saarinen L, Sara R, King AJ, Mooi FR, Soini JT, Mertsola J, He Q. Comparative genomics of Bordetella pertussis reveals progressive gene loss in Finnish strains. PLoS One. 2007;2(9):e904.

33. Parkhill J, Sebaihia M, Preston A, Murphy LD, Thomson N, Harris DE, Holden MT, Churcher CM, Bentley SD, Mungall KL, Cerdeño-Tárraga AM. Comparative analysis of the genome sequences of Bordetella pertussis, Bordetella parapertussis and Bordetella bronchiseptica. Nat Genet. 2003; 35(1):32.

34. Octavia S, Maharjan RP, Sintchenko V, Stevenson G, Reeves PR, Gilbert GL, Lan R. Insight into evolution of Bordetella pertussis from comparative genomic analysis: evidence of vaccine-driven selection. Mol Biol Evol. 2010; 28(1):707-15.
35. Mooi FR, Zeddeman A, Gent MV. The pertussis problem: classical epidemiology and strain characterization should go hand in hand. J Pediatr. 2015;91(4):315-7.

36. Mooi FR, Hallander H, Von König CW, Hoet B, Guiso N. Epidemiological typing of Bordetella pertussis isolates: recommendations for a standard methodology. Eur J Clin Microbiol Infect Dis. 2000;19(3):174-81.

37. Bahmanjeh A, Noofeli M, Khaki P, Hassanzadeh SM. Genetic analysis of clinical and vaccine strains of Bordetella pertussis by pulsed-field gel electrophoresis (PFGE), multi locus sequence typing (MLST) and serotyping. Comp Immunol Microbiol Infect Dis. 2019;64:168-75.

38. Preston A, Parkhill J, Maskell DJ. The bordetellae: lessons from genomics. Nat Rev Microbiol. 2004;2(5):379.

39. Vashishtha VM, Bansal CP, Gupta SG. Pertussis vaccines: position paper of Indian academy of pediatrics (IAP). Indian Pediatr. 2013;50(11):1001-9.

40. Lahariya C. A brief history of vaccines \& vaccination in India. Indian J Med Res. 2014;139(4):491

41. Jadhav S, Gautam M, Gairola S. Role of vaccine manufacturers in developing countries towards global healthcare by providing quality vaccines at affordable prices. Clin Microbiol Infect. 2014;20:37-44.

42. Weigand MR, Peng Y, Loparev V, Johnson T, Juieng P, Gairola S, Kumar R, Shaligram U, Gowrishankar R, Moura H, Rees J. Complete genome sequences of four Bordetella pertussis vaccine reference strains from serum Institute of India. Genome Announc. 2016;4(6):e01404-16.

43. Alai S, Ghattargi VC, Gautam M, Dhotre DP, Patel K, Pawar SP, Kumar R, Shaligram U, Deobagkar D, Gairola S. Genome sequence of Bordetella pertussis vaccine strain BP 165. Microbiol Resour Announc. 2019;8(16): e00150-19.

44. Sharma NC, Anandan S, Ragupathi NK, Sethuvel DP, Vasudevan K, Kumar D, Gupta SK, Sangal L, Murugesan V, Veeraraghavan B. Genetic diversity of clinical Bordetella pertussis ST2 strains in comparison with vaccine reference strains of India. bioRxiv. 2019;1:573543.

45. Bouchez V, Guglielmini J, Dazas M, Landier A, Toubiana J, Guillot S, Criscuolo A, Brisse S. Genomic sequencing of Bordetella pertussis for epidemiology and global surveillance of whooping cough. Emerging infectious diseases. 2018 Jun;24(6):988

46. Weigand MR, Peng Y, Loparev V, Batra D, Bowden KE, Burroughs M, Cassiday PK, Davis JK, Johnson T, Juieng P, Knipe K. The history of Bordetella pertussis genome evolution includes structural rearrangement. J Bacteriol. 2017;199(8):e00806-16.

47. Bowden KE, Weigand MR, Peng Y, Cassidy PK, Sammons S, Knipe K, Rowe LA, Loparev V, Sheth M, Weening K, Tondella ML. Genome structural diversity among 31 Bordetella pertussis isolates from two recent US whooping cough statewide epidemics. mSphere. 2016;1(3):e00036-16.

48. King AJ, van Gorkom T, van der Heide HG, Advani A, van der Lee S. Changes in the genomic content of circulating Bordetella pertussis strains isolated from the Netherlands, Sweden, Japan and Australia: adaptive evolution or drift? BMC Genomics. 2010;11(1):64.

49. Oliver A, Kay M, Cooper KK. Comparative genomics of cocci-shaped Sporosarcina strains with diverse spatial isolation. BMC Genomics. 2018; 19(1):310.

50. Chaudhari NM, Gupta VK, Dutta C. BPGA-an ultra-fast pan-genome analysis pipeline. Sci Rep. 2016;6:24373.

51. Tettelin H, Riley D, Cattuto C, Medini D. Comparative genomics: the bacterial pan-genome. Curr Opin Microbiol. 2008;11(5):472-7.

52. Park J, Zhang Y, Buboltz AM, Zhang X, Schuster SC, Ahuja U, Liu M, Miller JF, Sebaihia M, Bentley SD, Parkhill J. Comparative genomics of the classical Bordetella subspecies: the evolution and exchange of virulence-associated diversity amongst closely related pathogens. BMC Genomics. 2012;13(1):545.

53. Moran NA, Plague GR. Genomic changes following host restriction in bacteria. Curr Opin Genet Dev. 2004;14(6):627-33.

54. Achtman M. Insights from genomic comparisons of genetically monomorphic bacterial pathogens. Philos Trans R Soc Lond B Biol Sci. 2012; 367(1590):860-7.

55. Bouchez V, Caro V, Levillain E, Guigon G, Guiso N. Genomic content of Bordetella pertussis clinical isolates circulating in areas of intensive children vaccination. PLoS One. 2008;3(6):e2437.

56. Amman F, D'Halluin A, Antoine R, Huot L, Bibova I, Keidel K, Slupek S, Bouquet $\mathrm{P}$, Coutte L, Caboche S, Locht C. Primary transcriptome analysis reveals importance of IS elements for the shaping of the transcriptional landscape of Bordetella pertussis. RNA Biol. 2018;15(7): 967-75. 
57. Weigand MR, Williams MM, Peng Y, Kania D, Pawloski LC, Tondella ML, CDC Pertussis Working Group. Genomic Survey of Bordetella pertussis Diversity, United States, 2000-2013. Emerg Infect Dis. 2019;25(4):780.

58. Klimenko Al, Matushkin YG, Kolchanov NA, Lashin SA. Bacteriophages affect the evolution of bacterial communities in spatially distributed habitats: a simulation study. BMC Microbiol. 2016;16(1):S10.

59. Arndt D, Grant JR, Marcu A, Sajed T, Pon A, Liang Y, Wishart DS. PHASTER: a better, faster version of the PHAST phage search tool. Nucleic Acids Res. 2016;44(W1):W16-21.

60. Tiwari T, Murphy TV, Moran J. Recommended antimicrobial agents for the treatment and postexposure prophylaxis of pertussis: 2005 CDC guidelines. MMWR Recomm Rep. 2005;54(14):1-6.

61. Guillot S, Descours G, Gillet Y, Etienne J, Floret D, Guiso N. Macrolideresistant Bordetella pertussis infection in newborn girl, France. Emerg Infect Dis. 2012;18(6):966.

62. Wang Z, Cui Z, Li Y, Hou T, Liu X, Xi Y, Liu Y, Li H, He Q. High prevalence of erythromycin-resistant Bordetella pertussis in Xi'an, China. Clin Microbiol Infect. 2014;20(11):0825-30.

63. Xu Z, Wang Z, Luan Y, Li Y, Liu X, Peng X, Octavia S, Payne M, Lan R, Genomic epidemiology of erythromycin-resistant Bordetella pertussis in China. Emerg Microbes Infect. 2019:8(1):461-70.

64. Cummings CA, Brinig MM, Lepp PW, Van De Pas S, Relman DA. Bordetella species are distinguished by patterns of substantial gene loss and host adaptation. J Bacteriol. 2004;186(5):1484-92.

65. Kim SH, Lee J, Sung HY, Yu JY, Kim SH, Park MS, Jung SO. Recent trends of antigenic variation in Bordetella pertussis isolates in Korea. J Korean Med Sci. 2014:29(3):328-33.

66. Kallonen T, He Q. Bordetella pertussis strain variation and evolution postvaccination. Expert Rev Vaccines. 2009;8(7):863-75.

67. Sealey KL, Harris SR, Fry NK, Hurst LD, Gorringe AR, Parkhill J, Preston A. Genomic analysis of isolates from the United Kingdom 2012 pertussis outbreak reveals that vaccine antigen genes are unusually fast evolving. J Infect Dis. 2014;212(2):294-301.

68. Wagner B, Melzer H, Freymüller G, Stumvoll S, Rendi-Wagner P, Paulke-Korinek M, Repa A, Mooi FR, Kollaritsch H, Mittermayer H, Kessler HH. Genetic variation of Bordetella pertussis in Austria. PloS one. 2015;10(7):e0132623.

69. Borisova O, Kombarova SY, Zakharova NS, van Gent M, Aleshkin VA, Mazurova I, Mooi FR. Antigenic divergence between Bordetella pertussis clinical isolates from Moscow, Russia, and vaccine strains. Clin Vaccine Immunol. 2007;14(3):234-8

70. Kurova N, Njamkepo E, Brun D, Tseneva G, Guiso N. Monitoring of Bordetella isolates circulating in Saint Petersburg, Russia between 2001 and 2009. Res Microbiol. 2010;161(10):810-5.

71. Mosiej E, Augustynowicz E, Zawadka M, Dąbrowski W, Lutyńska A. Strain variation among Bordetella pertussis isolates circulating in Poland after 50 years of whole-cell pertussis vaccine use. J Clin Microbiol. 2011;49(4):1452-7.

72. Moriuchi T, Vichit O, Vutthikol Y, Hossain MS, Samnang C, Toda K, Grabovac V, Hiramatsu Y, Otsuka N, Shibayama K, Kamachi K. Molecular epidemiology of Bordetella pertussis in Cambodia determined by direct genotyping of clinical specimens. Int J Infect Dis. 2017;62:56-8.

73. Plješa $T$, He Q, Dakić G, Vignjević-Krastavčević Ml, Miković N, Ćirković I. Changes in genetic diversity of the Bordetella pertussis population in Serbia between 1953 and 2011. Arch Biol Sci. 2014;66(1):107-16.

74. Wattam AR, Abraham D, Dalay O, Disz TL, Driscoll T, Gabbard JL, Gillespie J, Gough R, Hix D, Kenyon R, Machi D. PATRIC, the bacterial bioinformatics database and analysis resource. Nucleic Acids Res. 2013;42(D1):D581-91.

75. Li L, Stoeckert CJ, Roos DS. OrthoMCL: identification of ortholog groups for eukaryotic genomes. Genome Res. 2003;13(9):2178-89.

76. Siguier $P$, Pérochon J, Lestrade L, Mahillon J, Chandler M. ISfinder: the reference centre for bacterial insertion sequences. Nucleic Acids Res. 2006; 34(suppl_1):D32-6

77. Bertelli C, Laird MR, Williams KP, Simon Fraser University research computing group, Lau BY, Hoad G, Winsor GL, Brinkman FS. IslandViewer 4: expanded prediction of genomic islands for larger-scale datasets. Nucleic Acids Res. 2017:45(W1):W30-5.

78. Tatusova T, DiCuccio M, Badretdin A, Chetvernin V, Nawrocki EP, Zaslavsky L, Lomsadze A, Pruitt KD, Borodovsky M, Ostell J. NCBI prokaryotic genome annotation pipeline. Nucleic Acids Res. 2016:44(14):6614-24.

79. Zankari E, Hasman H, Cosentino S, Vestergaard M, Rasmussen S, Lund O, Aarestrup FM, Larsen MV. Identification of acquired antimicrobial resistance genes. J Antimicrob Chemother. 2012;67(11):2640-4.
80. Grissa I, Vergnaud G, Pourcel C. CRISPRFinder: a web tool to identify clustered regularly interspaced short palindromic repeats. Nucleic Acids Res. 2007;35(suppl_2):W52-7.

81. Petkau A, Stuart-Edwards M, Stothard P, Van Domselaar G. Interactive microbial genome visualization with GView. Bioinformatics. 2010;26(24): 3125-6.

82. Darling AC, Mau B, Blattner FR, Perna NT. Mauve: multiple alignments of conserved genomic sequence with rearrangements. Genome Res. 2004; 14(7):1394-403.

83. Katoh K, Rozewicki J, Yamada KD. MAFFT online service: multiple sequence alignment, interactive sequence choice and visualization. Brief Bioinform. 2017;20:1160-6

84. Letunic I, Bork P. Interactive tree of life (iTOL): an online tool for phylogenetic tree display and annotation. Bioinformatics. 2006;23(1):127-8.

85. Choo V. Erythromycin-resistant Bordetella pertussis. Lancet. 1994;344(8936): 1564.

86. Safarchi A, Octavia S, Nikbin VS, Lotfi MN, Zahraei SM, Tay CY, Lamichhane B, Shahcheraghi F, Lan R. Genomic epidemiology of Iranian Bordetella pertussis: 50 years after the implementation of whole-cell vaccine. Emerg Microbes Infect. 2019:8(1):1416-27.

87. Aziz RK, Bartels D, Best AA, DeJongh M, Disz T, Edwards RA, Formsma K, Gerdes S, Glass EM, Kubal M, Meyer F. The RAST server: rapid annotations using subsystems technology. BMC Genomics. 2008;9(1):75.

\section{Publisher's Note}

Springer Nature remains neutral with regard to jurisdictional claims in published maps and institutional affiliations.
Ready to submit your research? Choose BMC and benefit from:

- fast, convenient online submission

- thorough peer review by experienced researchers in your field

- rapid publication on acceptance

- support for research data, including large and complex data types

- gold Open Access which fosters wider collaboration and increased citations

- maximum visibility for your research: over $100 \mathrm{M}$ website views per year

At $\mathrm{BMC}$, research is always in progress.

Learn more biomedcentral.com/submissions 